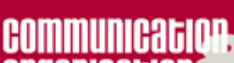
Organlofidin.

\section{Communication et organisation}

Revue scientifique francophone en Communication organisationnelle

\section{$24 \mid 2004$}

Coexister dans les mondes organisationnels

\title{
Les métiers de la communication en organisation. Le rôle des professionnels de la communication interne dans la gestion du lien social
}

Laura Salamanca Avila

\section{OpenEdition}

Édition électronique

URL : https://journals.openedition.org/communicationorganisation/2889

DOI : 10.4000/communicationorganisation.2889

ISSN : $1775-3546$

Éditeur

Presses universitaires de Bordeaux

Édition imprimée

Date de publication : 1 mai 2004

ISSN : 1168-5549

Référence électronique

Laura Salamanca Avila, « Les métiers de la communication en organisation. Le rôle des professionnels de la communication interne dans la gestion du lien social », Communication et organisation [En ligne], 24 | 2004, mis en ligne le 27 mars 2012, consulté le 21 septembre 2021. URL : http://journals.openedition.org/communicationorganisation/2889; DOI : https://doi.org/10.4000/ communicationorganisation.2889

Ce document a été généré automatiquement le 21 septembre 2021.

(C) Presses universitaires de Bordeaux 


\title{
Les métiers de la communication en organisation. Le rôle des professionnels de la communication interne dans la gestion du lien social
}

\author{
Laura Salamanca Avila
}

\section{Introduction}

1 L'objet de cet article est de comprendre, à partir d'une perspective communicationnelle et de l'analyse d'un cas, la manière dont les acteurs organisationnels de la communication, et plus précisément les responsables de la communication interne (CI.), gèrent les tensions au centre desquelles ils se trouvent avec un statut, en tant que médiateurs, en tant que porte-parole du sommet stratégique, et/ou en tant que porteparole de la communauté d'acteurs organisationnels.

2 Pour commencer, nous développerons brièvement les concepts de base de la réflexion tels que «culture d'organisation" et «communication interne». Ces concepts nous permettront de nous placer sur l'axe communicationnel à partir duquel nous nous centrerons, dans un deuxième temps, sur les métiers de la communication. À ce niveau, les concepts de légitimation et de légitimité seront précisés, ainsi que la définition des métiers de la communication interne et leur lien avec les processus de légitimation. Nous présenterons l'hypothèse d'analyse, qui nous permettra de localiser notre attention, dans la troisième partie, sur les fonctions des métiers de la communication.

3 Un classement des fonctions de communication en organisation sera présenté. Il se fonde sur l'étude de quelques organisations, ayant leur siège en Belgique. Ensuite, nous attirerons l'attention du lecteur sur quelques caractéristiques des métiers de la communication interne et sur le paradoxe occultation dévoilement du métier. Pour finir, 
nous formulerons quelques conclusions nous permettant de comprendre et de définir le rôle des professionnels de la $\mathrm{CI}$, sur le plan de la gestion du lien social en organisation.

\section{L'approche communicationnelle des organisations}

Dans ce premier point, nous proposons, d'une part, une définition de la culture organisationnelle et, d'autre part, de la communication interne.

Pour ce faire, nous basons notre réflexion sur une approche communicationnelle sur laquelle se fonde ce que l'on appelle depuis quelque temps «l'école de Louvain-la-Neuve» soutenue par quelques membres du Laboratoire d'Analyse des Systèmes de Communication d'Organisation (LASCO/UCL) de l'Université catholique de Louvain - P. de Saint-Georges., J. Pirson. V. Defourny. C. Duterme et nous-même. Cette approche se fonde sur l'école pragmatique de la communication (Waztlawick. Bateson. Selvini. Duterme, de SaintGeorges. Pirson), file constitue également une relecture, voire une prolongation communicationnelle des théories sociologiques des organisations qui, par leur apport, permettent de comprendre l'organisation en tant que fait societal, en tant que production collective d'identités (Sainsaulieu 1447), et en tant que système structuré par des enjeux stratégiques et des acteurs (Crozier et Friedherg 1477) vivant dans une permanente coopération conflictuelle.

\section{La culture organisationnelle}

Les notions "d'organisation », "d'interaction » et "d'interrelation » sont intimement liées (Morin. 1477). Le système constitue en effet une unité globale d'interactions entre ses membres. Les interactions qui y ont lieu, et qui sont régulées sont, dans ce sens, des unités de comportement, des éléments, qui additionnés ou mêlés à d'autres interactions, constituent des chaînes d'interactions.

7 Ainsi, l'ensemble des interactions qui se répètent constitue-t-il ce qu'on nomme des modèles de redondance (Watzlawick 1472). Les interactions sont donc au cœur de l'action collective car, d'une part, elles véhiculent des codes culturels et d'autre part, elles sont l'unité de référence pour l'analyse des comportements et des contextes organisationnels.

De ce fait, les modèles de redondance sont liés aux modes de régulation - il s'agit bien de deux phénomènes organisationnels interdépendants. Le modèle de redondance est constitué par les chaînes d'interactions qui se répètent, alors que le mode de régulation est relié à la manière dont se créent et se transforment les règles. Autrement dit, l'ensemble des modèles de redondance permet la mobilisation, la transformation, la disparition ou même l'émergence des nouvelles règles, donc des nouveaux modes de régulation.

9 Dans les organisations, nous distinguons la coexistence de deux grands types de codes culturels, les codes sociétaux et les codes propres à la culture de l'organisation. Premièrement, il existe des codes que l'acteur s'est approprié depuis sa naissance et auxquels il a été socialisé. Il s'agit des codes culturels sociétaux. Ces codes sont liés, par exemple, à l'appartenance à une société ou à une communauté.

10 Deuxièmement, dans les organisations, l'acteur intègre dans sa vie quotidienne une variété de codes culturels propres à son entourage organisationnel. Il s'agit non 
seulement des codes normatifs - ensemble de valeurs, croyances, représentations, chartes, procédures, manières d'agir et de penser en organisation -, mais également des codes comportementaux.

11 Pour que les modèles de redondance aient un sens, qui leur sera accordé par les partenaires de la communication, il nous faut les placer dans certain(s) contexte(s). Dans ce sens, à l'intérieur d'une organisation, qu'il s'agisse d'une famille, d'un groupe ou d'une entreprise, le sens du comportement des acteurs est donné par la manière dont les partenaires de la communication s'adaptent au(x) contexle(s).

12 C'est en fonction de l'apprentissage des codes culturels (Watzlawick 1472) que les partenaires d'une communication disposeront des éléments pour déterminer «ce au il convient de faire et ou de dire » dans un contexte déterminé. On ne peut donc pas parler d'un contexte mais de contextes, car l'organisation est constituée de sous ensembles. Le respect et la valorisation de la variété des contextes organisationnels constituent l'une des particularités de l'approche communicationnelle Je l'organisation.

Dans ce cadre, la culture d'organisation peut être considérée comme régulatrice de la communication interne, donc de l'action collective. Elle recouvre tous les acteurs organisationnels - avec leurs intérêts et leurs rapports conflictuels au niveau groupai et collectif. L'identité d'une organisation est portée par des codes culturels partagés dans une négociation de coopération conflictuelle par les membres de l'organisation.

L'analyse de la culture organisationnelle consiste donc, au sens large, en la compréhension de la nature des interactions qui s'y déroulent, tout en gardant un œil sur le(s) contexte(s) dans lequel elles ont lieu et sur les modes de régulation qui y règnent.

Dans ce cadre, l'analyse de la manière dont les différents niveaux ou hiérarchies de contextes s'entrelacent, nous permet de comprendre le sens collectif accordé aux interactions et aux codes culturels en organisation.

\section{La communication interne}

16 Par communication interne, nous comprenons l'ensemble des comportements et des modèles de redondance - survenus dans des contextes variés régulés par des codes culturels.

17 Le syntagme communication interne est utilisé de manière indistincte par des sociologues, par des professionnels de la gestion et de la communication et également, par des psychosociologues. Sans vouloir approfondir les différentes versions par rapport au sujet, nous voulons, pour situer la nôtre, en nommer quelques-unes (de Saint-Georges 2000. Giroux 1994 et Duterme 2002). à notre avis les plus représentatives.

L'approche opérationnelle de la communication première lecture des phénomènes de communication en organisation se centre sur la manière dont les messages sont véhiculés. Dans ce cadre, des auteurs parlent de communication interne pour décrire l'ensemble des dispositifs ou outils informant ainsi que les instructions, les ordres, les directives, etc. Ces dispositifs sont, bien évidemment, des aspects de la communication, mais ils ne revendiquent que l'aspect instrumental de la communication, c'est-à-dire l'information de commandement et de contrôle de gestion.

19 L'approche intégrative vient notamment de la prise en compte de l'importance du facteur humain dans la production. C'est ainsi que « le moral des troupes » et la motivation sont 
devenus des sujets largement traités depuis une trentaine d'années. Dans ce cadre, on entend par dimension intégrative de la communication l'ensemble des actions entreprises dans le but de motiver et de maintenir une certaine cohésion interne autour d'un certain nombre de valeurs et d'objectifs. Évidemment, cette approche soulève une question importante : celle de la reconnaissance collective autour d'un certain nombre de valeurs, de normes, de représentations. C'est dans ce cadre que l'on trouvera par exemple les revues, les journaux, les brochures, la presse d'entreprise, etc.

Lorsqu'on parle de communication interne, on se rend compte alors que les deux premières approches risquent de conduire à une confusion entre la communication organisationnelle (les comportements, les phénomènes, les interactions observées) et la communication d'organisation (les politiques menées pour influencer les comportements). Or, ces dernières ne révèlent qu'une partie de l'univers de significations de l'organisation et une volonté de « régulation de contrôle » au sens reynaudien (1997), donc de « communication contrôlée » de la part du sommet stratégique. Les politiques ou stratégies - de communication et d'information formulées par le management ou le sommet d'une organisation forment une partie de la communication interne. Mais elles ne sont, à nos yeux, qu'une dimension de celle-ci.

En conclusion, la communication organisationnelle interne n'est pas limitée au contrôle et à la gestion des aspects opérationnels, managériaux de la communication des membres d'une partie de l'organisation sur une autre. Nous prônons donc une définition explicative de la communication interne dans laquelle celle-ci est comprise comme l'ensemble contextualisé des comportements et des opinions en interaction. En ce sens, l'organisation est bien un système de communication

Nous avons ainsi résumé le contexte théorique à partir duquel nous allons aborder, maintenant, les métiers de la communication en organisation et leur rôle au niveau (du maintien) du lien social en organisation.

\section{Les métiers de la communication}

23 Si la communication interne est considérée comme un ensemble de comportements et d'opinions, l'exercice des métiers de la communication en organisation chercherait, d'un côté, à faire part de la diversité des contextes, des modes de régulation et des modèles de redondance de l'organisation et, d'un autre côté, tendrait vers une confrontation positive de ceux-ci afin de créer, d'assurer, voire d'établir une certaine cohésion ou un lien social organisationnel (faire le lien entre les différents contextes organisationnels), ainsi qu'un certain degré d'apprentissage organisationnel autour d'un certain nombre de codes culturels et de modes d'interaction partagés.

\section{Les concepts de légitimation et de légitimité}

Les légitimations sont, d'une part, des contenus "mentaux» de nature intellectuelle, morale, affective, sociale, de sens commun, etc. (Weber) et, d'autre part, les processus par lequel on arrive à légitimer les premiers. Ces contenus sont en relation avec les positions et les situations sociales des individus, des groupes, des organisations, et avec les normes et croyances que les divers groupes sociaux entretiennent dans les organisations et dans les diverses sphères de la société. 
Selon Weber « un pouvoir légitime est celui qui a la capacité de faire accepter ses décisions comme bien fondées, suscitant l'adhésion (enthousiaste) ou au moins l'acquiescement (résigné) des destinataires. Mais le fondement le plus sûr de la domination n'est pas tant la puissance que la croyance en la validité de la puissance. La croyance s'ajoute ou se substitue à la peur, lu poursuite rationnelle de l'intérêt ou la coutume pour expliquer la stabilité d'un ordre social: sur elle repose la légitimité de l'ordre ».

Dans ce cadre, la légitimation est un processus d'attribution du sens dans un rapport social où s'exerce un pouvoir. En même temps, elle est aussi un système d'explication, un argumentaire, un contenu mental.

Dans ce cadre, la légitimation est l'acte par lequel, lors d'une interaction, des contenus mentaux sont véhiculés en acquérant du sens - à l'échelon du groupe et/ou de la collectivité concernée. Ainsi, la légitimation d'un métier, outre de constituer un acte de pouvoir du professionnel (de compétitivité intellectuelle et empirique), est une manière, sur l'échelle organisationnelle de réguler le travail de ses membres qui sont liés à un métier et aux fonctions propres à celui-ci.

Néanmoins, l'exercice du pouvoir n'est autre qu'un acte de communication caractérise par la mise en place d'un rapport de forces, donc d'une relation stratégique. Cet aspect nous met sur la piste de la légitimité des métiers de la communication en organisation, comme faisant partie des enjeux de pouvoir, et donc du rapport de force. Ce cas se constaterait davantage dans les organisations de grande taille dans lesquelles la gestion des interactions se révèle plus complexe.

\section{Les métiers de l'information et de la communication en organisation}

29 En ce qui concerne les métiers de l'information et de la communication en organisation, une variété de professions et de fonctions liées à cette profession sont proposées par J.-L Michel (1999) et par C. Duterme (2002). Ces auteurs remarquent bien l'ambiguité apportée par la dénomination des métiers de la communication. En effet, les fonctions et les résultats de I exercice du métier de communicateur sont difficilement mesurables.

Ce flou, qui est un enjeu, tant pour l'organisation que pour ces professionnels, « empêche de parvenir à l'établissement de catégories et de hiérarchies professionnelles précises » (Michel. 1999).

1 Dans ce cadre, il est possible d'avoir autant de «fonctions » et de "professionnels » de l'INFOCOM que de structures et de contextes organisationnels. Cependant, trois niveaux principaux de l'exercice du métier sont présentés par J.-L. Michel, Le premier est le niveau stratégique dans lequel se place le DIR COM, le deuxième est le fonctionnel dans lequel se placent les chargés de communication et le troisième est l'opérationnel dans lequel se situent les rédacteurs, les éditeurs Web. Etc.

La tâche principale de ces professionnels de l'INFOCOM (Duterme. 2002 et Michel. 1999) serait de veiller à un équilibre entre "l'image » de l'organisation et son «identité ». Cet équilibre souhaité est possible par la transmission de l'information. Progressivement, les professionnels de la $\mathrm{CI}$, concentreront leur attention sur la perception de l'entreprise par le personnel et sur son image interne. 

" image positive» qui correspondrait aux souhaits des décideurs de l'organisation» (Duterme. 2002). CI serait - c'est notre hypothèse - non seulement un enjeu de pouvoir et de légitimation du discours managérial mais un enjeu lors d'une crise, d'un changement ou, en général, lors d'un moment critique dans lequel le rôle de médiation du communicateur pourrait être valorisé et prendre une place importante par rapport au rôle de canalisateur de l'information. Autrement dit, nous croyons que les fonctions, à tendance opérationnelle, exercées par les professionnels de la CI manquent, dans la plupart des cas, d'un volet stratégique et, par conséquent. D'une lecture communicationnelle de l'organisation et des phénomènes relationnels qui $\mathrm{j}$ ont lieu.

\section{Une analyse empirique des métiers de la communication} d'analyse de la communication institutionnelle dirigé à l'Université catholique de Louvain-laNeuve par J. Pirson (2001-2002). L'objectif du séminaire était l'analyse de la culture organisationnelle et des pratiques des professionnels de la communication interne.

Six organisations (du secteur marchand et non marchand), ayant un siège en Belgique, ont été retenues pour cette analyse - une vingtaine d'organisations avaient été étudiées dans le cadre du séminaire. classement des organisations analysées: le premier est la taille et la structure de l'organisation, le second est l'emplacement des professionnels de la communication interne au niveau de la structure (organigramme) de l'organisation et le dernier est la description du métier et des missions de la fonction de communication liées au(x) métier (s) de la communication. directement gérée, en interne, par des responsables de ce métier. Le positionnement hiérarchique des chargés de la $\mathrm{CI}$ était variable ainsi que leur niveau de prise de décision. L'objectif de cette étude était de répondre à la question suivante : En quoi les pratiques des professionnels de la communication interne révèlent-elles des indicateurs pour la compréhension du lien social en organisation? permettra de répondre à cette question

Classement des fonctions de communication en organisation.

\section{Conclusions de l'étude de cas}

42 La première conclusion, en lien avec l'existence d'un mandat explicite de CI., est liée à la configuration organisationnelle (Mintzberg 1998). En effet, les modèles sont liés à la taille et à la structure des organisations. Ainsi, dans une petite organisation (modèle I), aucune personne n'est-elle chargée de la communication car, d'une part, celle-ci semble avoir 
lieu spontanément et, d'autre part, les liens de proximité, tant physique qu'émotionnelle des employés garantissent la circulation de l'information entre ceux-ci.

Lorsque la taille de l'organisation est plus importante (modèle 2), des moyens sont destinés à l'exercice de quelques fonctions de la communication interne. Cependant, ces fonctions, étant réparties entre des professionnels qui d'ailleurs ne sont pas toujours experts en lu matière semblent être dispersées et manquent de coordination centrale.

Lorsque la taille de l'organisation est encore plus importante (modèle 3) comme c'est le cas des organisations ayant plusieurs sièges ou des entreprises multinationales, des moyens sont consacrés à la création d'un département de communication (interne et externe) qui centralise les différentes fonctions et qui veille, d'une manière plus soutenue et planifiée, à son bon fonctionnement.

Dans ce cadre, les professionnels de la communication, comme nous l'avons constaté, peuvent être des personnes avant suivi des formations d'ingénieur commercial, de communication, de psychologie, de journaliste ou de juriste, parmi d'autres.

Indépendamment de la taille et du type d'organisation, nous avons constaté que, lorsque le mandat de communication existe, dans la plupart des cas, il n'est pas posé en termes stratégiques, mais plutôt en termes opérationnels et/ou fonctionnels. Une autre caractéristique du mandat des responsables de la $\mathrm{CI}$ est liée à une souplesse vécue comme stressante et dangereuse du mandat en tant que tel. En effet, un cahier de charges précis est rarement fourni au nouvel arrivé. Ce n'est qu'avec le temps et l'expérience, acquise ailleurs ou dans la même organisation, que le professionnel parvient à légitimer un certain nombre de fonctions.

Cependant, dans la plupart des cas étudiés, le principal objectif explicite du métier de la $\mathrm{Cl}$. est l'information. Parmi les objectifs secondaires, et plus implicites, on retrouve la gestion de l'image. Un paradoxe est ici à l'œuvre. Il est lié, d'une part, à la demande implicite de gestion d'une bonne image de l'organisation et, d'autre part, à la demande explicite de transparence et d'impartialité dans l'exercice du métier. Dans ce cadre, il est démontré que la légitimation du professionnel de la $\mathrm{Cl}$. est un enjeu de pouvoir, d'une part, du professionnel lui-même. qui. se fondant sur sa formation, son expérience et son charisme, tente de valider un certain nombre de principes liés à l'exercice du métier et, d'autre part, des autres acteurs de l'organisation notamment du sommet stratégique qui verraient, dans l'exercice du métier, une occasion de transmettre une volonté politique de manipulation de l'information.

Cette confusion quant au rôle paradoxal d'occultation/dévoilement des professionnels de la CI, met en évidence, d'une part, l'ambiguïté autour de l'exercice du métier, qui serait un symptôme d'une certaine culture managériale et, d'autre part, la mise en valeur des compétences liées aux lâches à caractère opérationnel, qui prédomineraient sur les tâches liées à l'analyse du lien social dans l'organisation, passage obligé cependant pour la compréhension de la CI. et pour de développement de celle-ci.

Cette étude nous permet de démontrer que la légitimité des métiers de la CI. est en cause, ou devient même un enjeu, au point que pour les membres du sommet stratégique de l'organisation, le changement et/ou la crise pourraient servir de moteurs de légitimation ou de delégitimation des métiers de lu C.I., de même que de leurs mandats. Cette conclusion apparait complémentaire à la croyance des employés de l'organisation, répandue dans les cas étudiés, selon laquelle le travail du communicateur interne prendrait sa légitimité 
particulière en situation de crise, et serait moins perceptible, voire visible en situation stable.

\section{Conclusion : le lien social et les métiers de la communication}

Un constat observé lors de cette étude est le fait que pour la plupart des professionnels de la Cf. l'action sur le lien social consiste en le fait "d'informer tout le monde de la même manière». Cette affirmation nous permet de comprendre que le champ de la communication en organisation n'est pas encore un acquis et qu'une réflexion importante sur le rôle du professionnel de la CI mérite d'être approfondie. En effet, nous croyons que celui-ci consisterait, avant tout, dans le pilotage de la communication dans l'organisation, dans la garantie et l'encouragement du respect de lu différence, par la mise en place de différents outils d'information et de communication.

51 Ainsi, nous croyons que le principal enjeu des métiers de la CI. est de passer de la fonction d'exécutant à la fonction stratégique. Le professionnel est appelé à être un métacommunicateur capable de gérer le lien social en organisation.

Dans ce sens, l'objectif de la CI est de faire en sorte que les différents intérêts cohabitent car il ne faut pas laisser au hasard l'adoption des conduites adéquates, donc des modèles de redondance et des modes de régulation en organisation.

Le professionnel de la CI serait dans ce sens appelé à favoriser l'existence et le respect des sous-cultures car comme nous l'avons expliqué dans la première partie, il n'y a pas une culture, il y a des sous-cultures, des manières différentes de voir et de vivre, par le biais des interactions, la réalité de l'organisation.

Dans ce sens, les professionnels de la CI seraient également appelés à faire part de la « coopération conflictuelle » (Sainsaulieu. 1997) donc de l'existence d'un certain dialogue, d'une concertation collective qui dépasse les luttes de pouvoir» des acteurs, et qui permet de faire émerger le sens collectif du lien social.

55 Ainsi, selon notre approche, la gestion des modèles de redondance et des modes de régulation n'est autre que la manière dont, à travers la gestion de la coopération conflictuelle, les acteurs parviennent à vivre le lien social, à construire et reconstruire le système de codes culturels et à choisir des manières de les véhiculer : permettant l'émergence de nouveaux apprentissages et d'une identité organisationnelle propre qui serait ainsi cohérente avec «l'image transmise ».

Le rôle des professionnels de la CI serait en somme de respecter la pluralité d'opinion, garantissant le maintien de la diversité du processus d'apprentissage dont l'organisation a besoin pour survivre et pour s'accroître. En même temps il est appelé à maintenir une identité commune, respectueuse des modes de régulation globaux et des modèles de redondance locaux, qui sont, en effet, des repères comportementaux qui déterminent la communication interne.

57 Se fondant sur une stratégie de communication, le communicateur pourra adapter les outils nécessaires au maintien du lien social, au développement de la culture et de la reconnaissance collectives qui font vivre le lien social et qui l'ont que l'organisation évolue, en même temps que ses acteurs. 


\section{BIBLIOGRAPHIE}

BATESON G. I ers une écologie de l'esprit I. Paris : Seuil Seuil. 1997.

BERNARD'. S. PIRSON. J., SALAMANCA I... Pratiques professionnelles de communication : différenciation et légitimation. In Journée d'études annuelles. Groupe de contact du fonds National de la Recherche Scientifique Belge (FNRS). décembre 2002. Bruxelles.

DE SAINT-GEORGES P., Culture d'entreprise, communication interne et stratégie de changement. Revue Communication et Organisation : les relations publiques face à la théorie. $\mathrm{N}^{\circ} 4$. GREC/O. ISIC. Université Michel de Montaigne 3. Bordeaux, nov. 1993. pp. 71-93.

DL SAINT-GEORGES P., La « formation-symptôme ». Intervention et changement dans les organisations. Revue Communication et Organisation. № 17. $2^{\mathrm{e}}$ semestre. GREC/O. [SIC, Université Michel-de-Montaigne 3. Bordeaux. 2000. pp. 195-218.

DUTERME C. La communication interne en entreprise : l'approche de Palo Alto el I analyse des organisations. Bruxelles : De Boeck Université. 2002. GIROUX N., La communication interne : une définition en évolution. Revue Communication et organisation. Bordeaux n 5. 07/1994 GREC/O. ISIC. pp. 17-45.

MICHEL.J.-I... Les professions de la communication : fonctions et métiers. Paris : Ellipses, collection Infocom. 1999.

MINTZBERG H. Structure et dynamique des organisations. Paris : Editions d'organisation. 12 ème tirage. 1998.

MORINE., La méthode. Tome I : la nature de la nature. Paris : Éditions du Seuil. Collection « Points politiques ». 1977.

PIRSON.J.. Changer : s'adapter ou innover. Pour une lecture communicationnelle des organisations culturelles. Thèse en sciences sociales (information et communication). Louvain-la-Neuve : Département de Communication, faculté de sciences économiques, sociales et politiques. Université Catholique de Louvain. 1998.

REYNAUD J.-D., Les règles du jeu : l'action collective et la régulation sociale. Paris : Armand Colin. $3^{\text {ème }}$ édition. 1997.

SAINSAULIEU R., Sociologie de l'entreprise, organisation, culture et développement. Mayenne : Presses de Sciences Politiques et Dalloz 1997. SELVINI PALAZZOLI (éd.). Dans les coulisses de l'organisation. Paris : Éditions I SI. 1984. 


\section{AUTEUR}

\section{LAURA SALAMANCA AVILA}

Université catholique de Louvain-la-Neuve, Belgique. Laboratoire d'analyse des systèmes de communication d'organisation (LASCO/UCL) 